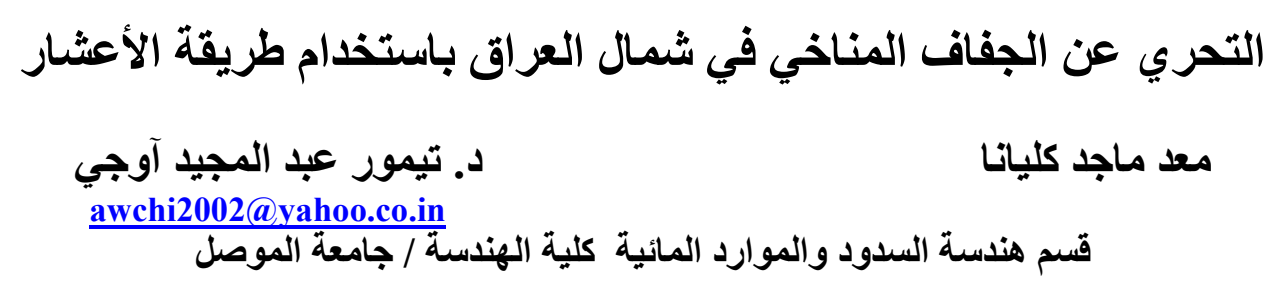

\title{
الخلاصة
}

تعتبر طريقة الأعشار Deciles واحدة من الدائل التي تستخدم في دراسة وتحليل الجفاف في مختلف أنحاء

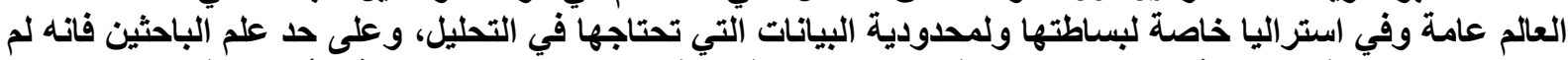

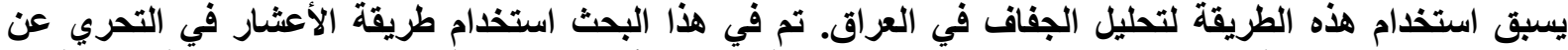

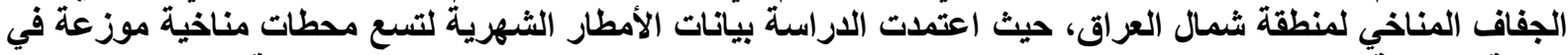

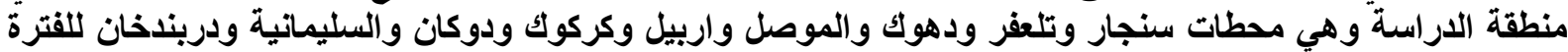

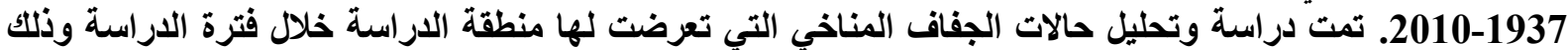

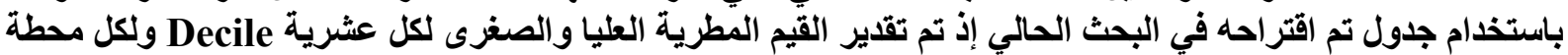

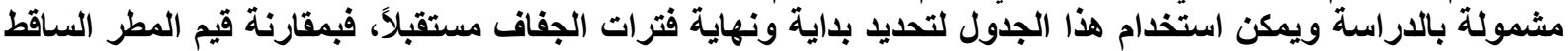

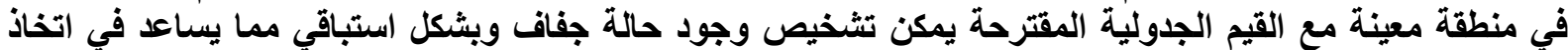

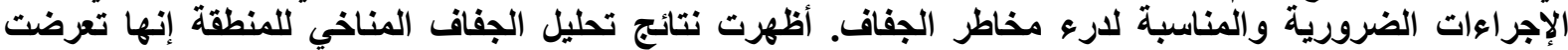

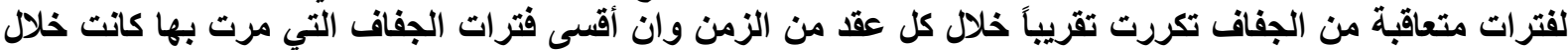
الفترة 2001-1997 و2010-2007.

\section{Investigating the Meteorological Drought in Northern Iraq Using Deciles Method}

\section{Maad Majid Kalyana}

\author{
Dr. Taymoor A. Awchi
}

Deciles method is one of the indices which are used for studying and analysing of droughts in the world and especially in Australia. The method is known by its simplicity and the limited data needed for analysis, and up to the knowledge of the authors this paper is the first study which utilizes this method for drought analysis in Iraq. Deciles method is used in this research for meteorological drought investigation in Northern Iraq. Monthly rainfall data is collected and utilized from 9 meteorological stations scattered in the study area which are; Sinjar, Tel-Afer, Dohuk, Mosul, Erbil, Kirkuk, Dokan, Sulymania, and Derbendikhan for the period 1937-2010. The meteorological drought cases were studied and analyzed using a table proposed in this study. The table includes the proposed maximum and minimum rainfall depths for each decile and for each station involved in the study. This table can be used to identifying drought periods' set on and termination in future. This can be achieved by comparing the actual rainfall depths in a certain area with the proposed tabulated values to diagnose drought cases which help in taking suitable actions for drought mitigation in advance. The drought analysis for the study area showed that the area has faced a sequential drought periods which were recurring about every decade and the most severe drought periods were in 1997-2001 and 2007-2010.

Keywords: Deciles Method, Meteorological Drought, North of Iraq

$$
\text { قبل: } 2015 \text { - } 4 \text { - } 21
$$

أستلم: 2013 - 9 - 23 
المقدمة

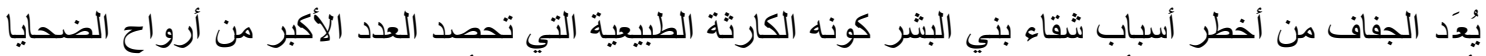

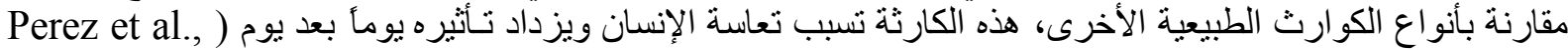

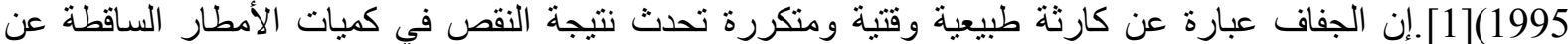
معدلاتها الطبيعية وتجلب خسائر اقتصادية بالغة التأثثر، و هذا يحدث بسبب حسبة حصول خلل أو عدم توازن في في أو اصر الدورة

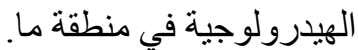

يمكن وصف الجفاف على انه دمار بطىئ الزحف، لا يمكن تفاديه ولكن بمكن تطوير إمكانات التهيؤ له وقائه وقابلية

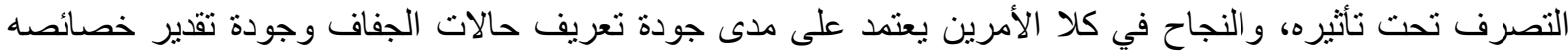

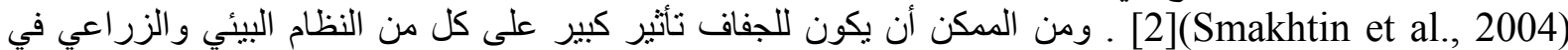

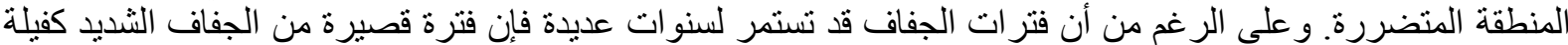
بإلحاق أضر ار و إنز ال خسائر هائلة بالاقتصناد المحلي.

هنالك عدة أنواع ومر احل للجفاف والتي تعبر عن وجهة النظر لمختلف أنواع النقص في المياه مثل الجفاف المناخي

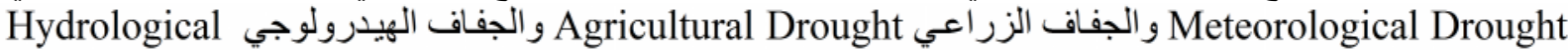

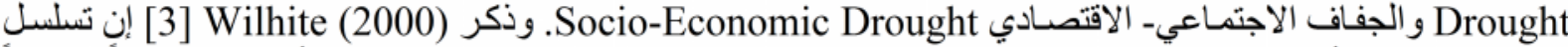

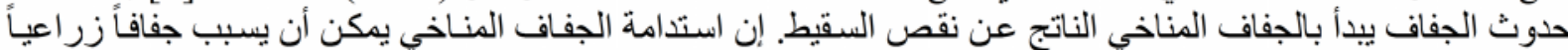

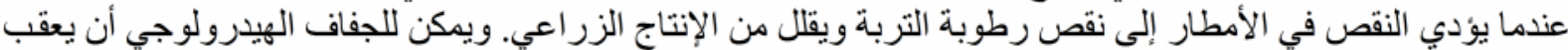

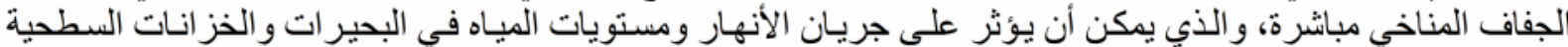

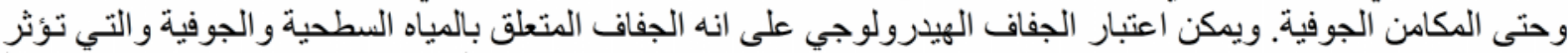

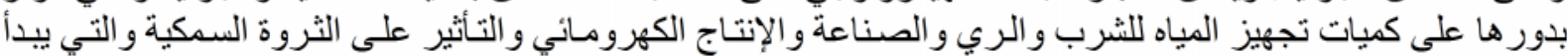

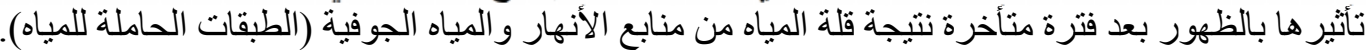

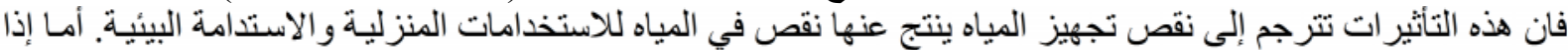

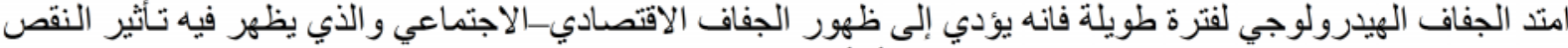

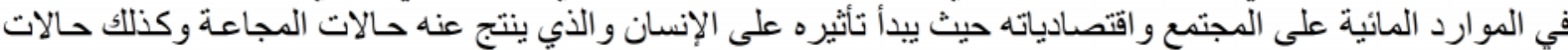

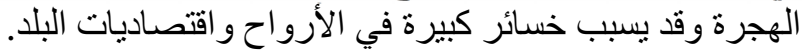

يعتمد الباحثون و المختصون عددا من الدلائل Drought Indices لدر اسة الجفاف وتحليله بنوع و عدد المتغيرات التي تحتاجها في التطبيق فضلا عن الغرض الذي الذي يستخدم من اجلها. من أهم هذه الدلائل

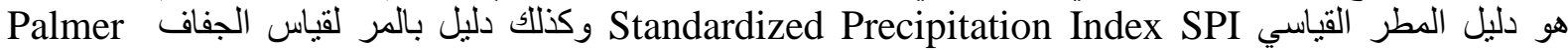
Deciles وطريقة العشريات Drought Index PDI في استر اليا تعتمد طريقة Deciles كإحدى طر ائق قياس الجفاف في نظام مر اقبة الجفاف الاستر الي (ADWS)

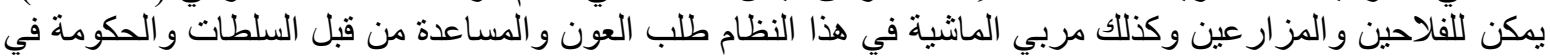

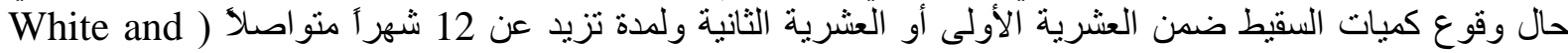

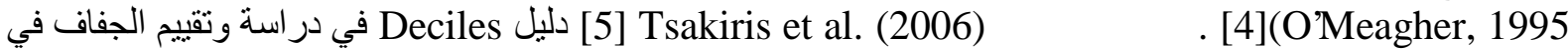
حوضي نهر (Moruos) ونهر (Nestos) في اليونان. حيث استخدمت بيانات الأمطار ودرجات الحرارة الثهرية لعشرة [6] Timbal (2007)

- منرق استر اليا باستخدام طريقة Deciles. .2006-1962

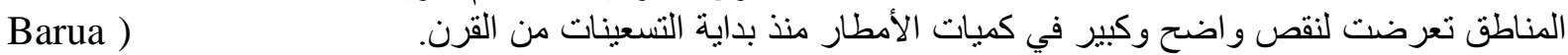

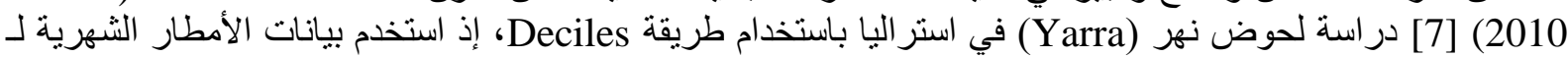
.2008-1960

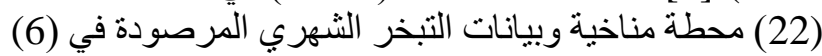

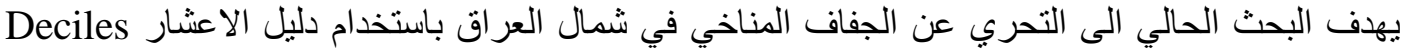

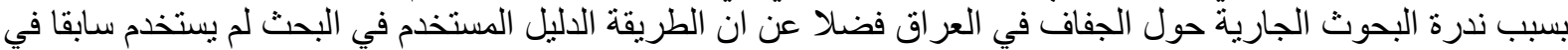

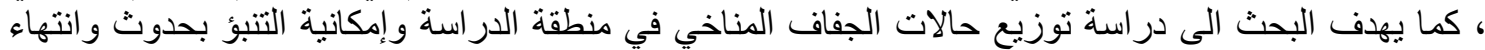

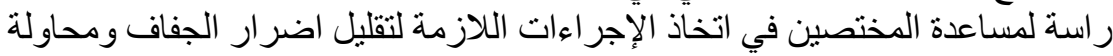


63355

\section{منطقة الدراسة}

الثمالية والثرقية إذ تفصلها عن الأر اضي التركية والإير انية على التو الي.

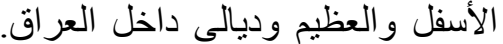

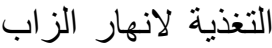

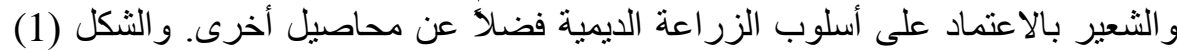

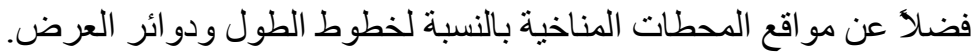

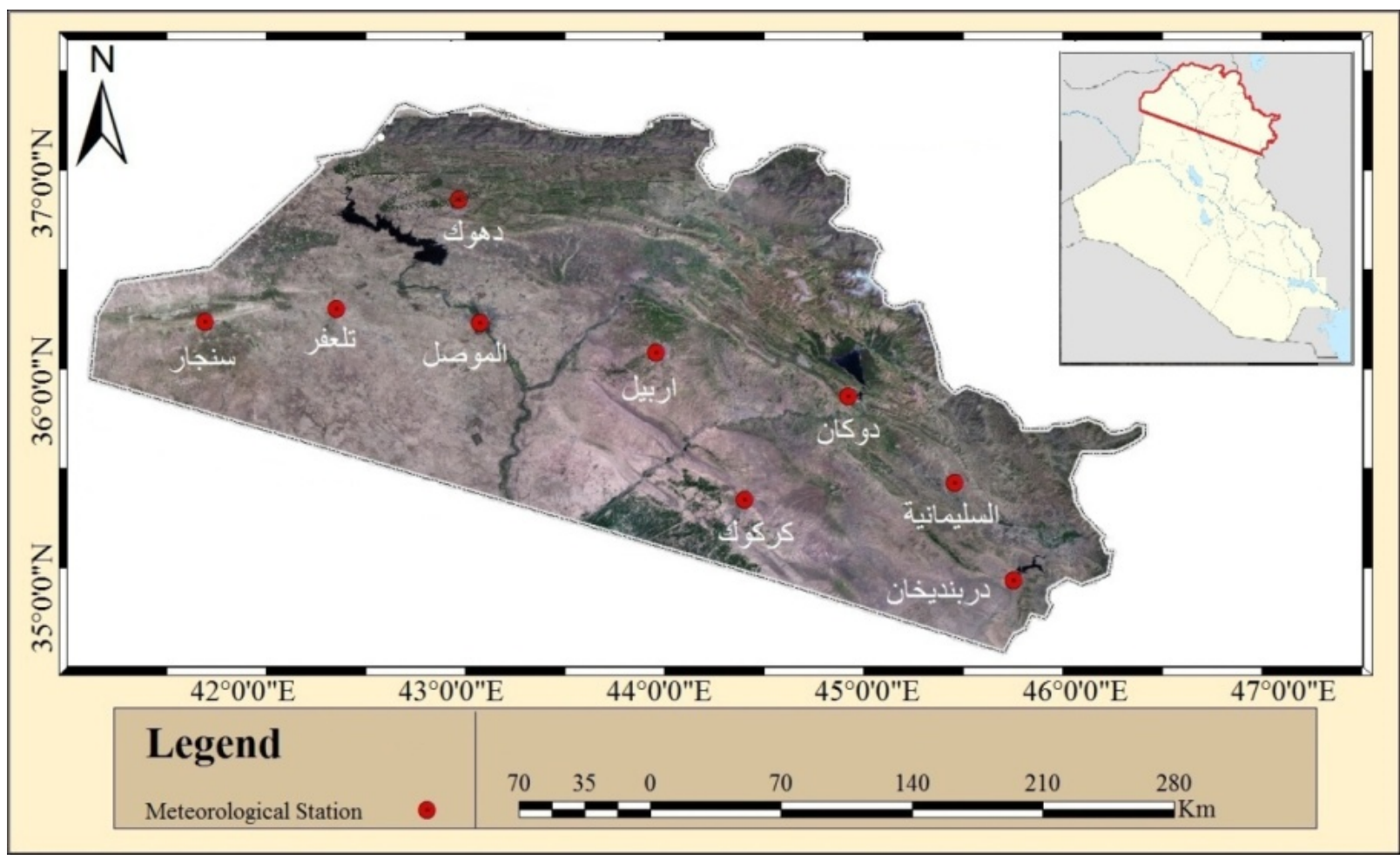

(1): خارطة منطقة الدر اسة موضح عليها المحطات المناخية المختارة

(1): التحليل الإحصائي وبعض خصائص السجلات المطرية للمحطات المختارة ضمن منطقة الدر اسة

\begin{tabular}{|c|c|c|c|c|c|c|c|}
\hline 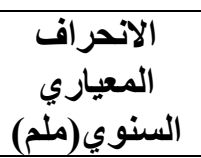 & 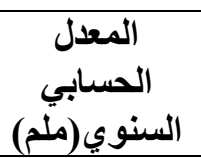 & 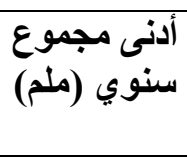 & سنوي (ملم) & الفترة & المطري السنةل) & المحطة & $ت$ \\
\hline 226 & 767 & 366 & 1468 & 2010-1945 & 66 & & 1 \\
\hline 192 & 700 & 339 & 1244 & 2010-1941 & 70 & السليمانية & 2 \\
\hline 200 & 675 & 296 & 1235 & 2010-1962 & 49 & دربنديخان & 3 \\
\hline 170 & 570 & 225 & 911 & 2010-1943 & 68 & دهوك & 4 \\
\hline 141 & 431 & 157 & 886 & 2010-1941 & 70 & اربيل & 5 \\
\hline 110 & 378 & 194 & 633 & 2010-1937 & 74 & & 6 \\
\hline 138 & 378 & 164 & 670 & 2010-1941 & 70 & & 7 \\
\hline 129 & 367 & 135 & 770 & 2010-1940 & 71 & & 8 \\
\hline 108 & 321 & 134 & 614 & 2010-1941 & 70 & & 9 \\
\hline
\end{tabular}


تم الحصول على بيانات الأمطار الثهرية المسجلة في تسع من محطات الأنواء الجوية في شمال العراق ودوات وهي

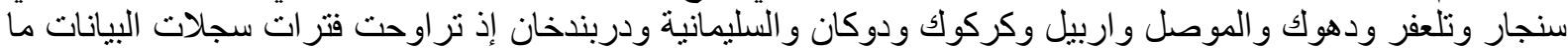

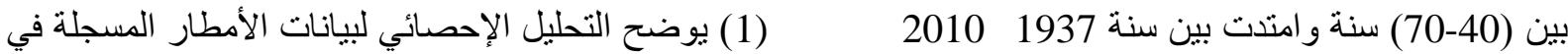

تعاني سجلات اغلب محطات الأنواء الجوية العراقية من فقدان البيانات التي قد تصل لعدة ألثات أشهر أو عدة سنوات،

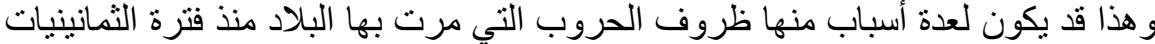

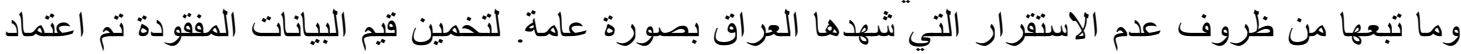

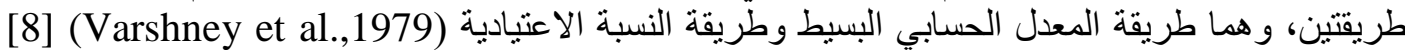
تعتمد هاتان الطريقتان على معدلات السقيط الثهرية للمحطات المجاورة للمحطة المعنية.

\section{Deciles}

دليل الأعشار

ثُعَد هذه الطريقة من الطر ائق السهلة الاستخدام نسبياً والتي تحتاج إلى بيانات الأمطار فقط واقترحت من قبل

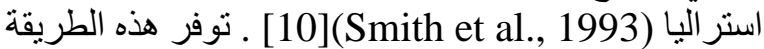

[9] Gibbs and Maher (1967)

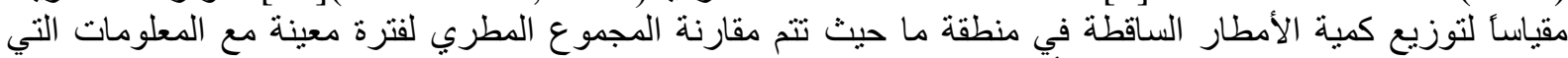

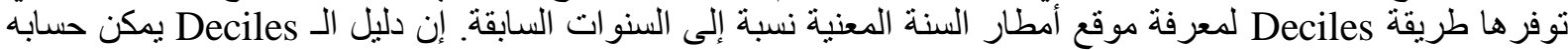

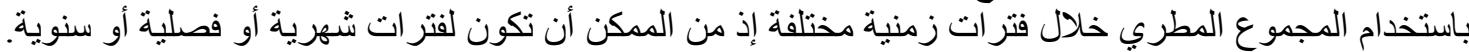

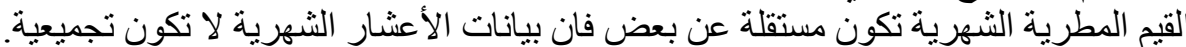

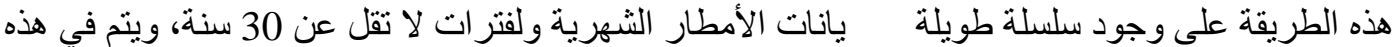

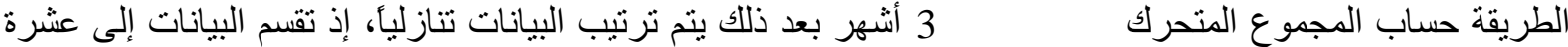

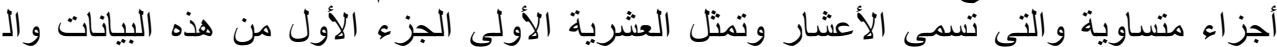

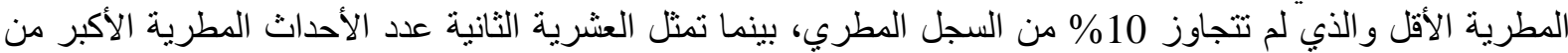

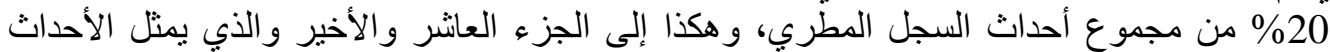

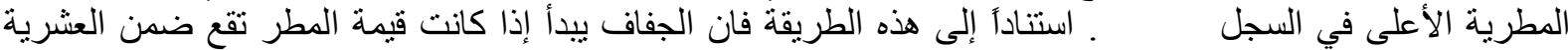

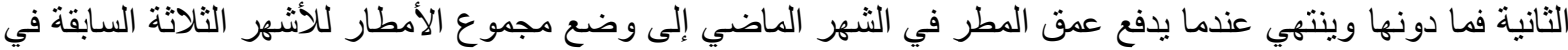

[9] Gibbs and Maher (1967) . [11](Kinninmonth et al., 2002) العشربة إلى خمسة أصناف مختلفة وكما هو موضح في الجدول (2).

Deciles (2): تصنيف الجفاف بحسب طريقة الأعثار

\begin{tabular}{|l|c|}
\hline \multicolumn{1}{|c|}{ Deciles الأعشار } & التصنيف| \\
\hline Deciles 1-2 (lowest 20\%) & Much below normal \\
\hline Deciles 3-4 (next lowest 20\%) & Below normal \\
\hline Deciles 5-6 (middle 20\%) & Near normal \\
\hline Deciles 7-8 (next highest 20\%) & Above normal \\
\hline Deciles 9-10 (highest 20\%) & Much above normal \\
\hline
\end{tabular}

ايجابيات طريقة Deciles أنها سهلة الحسابات ولكن سهولتها قد تؤدي إلى صعوبات مفاهيمية، على سبيل

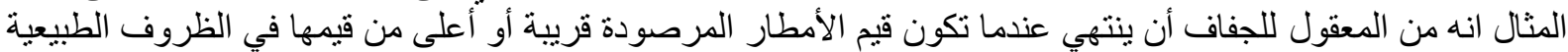

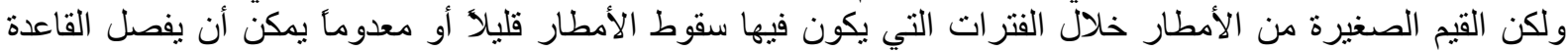

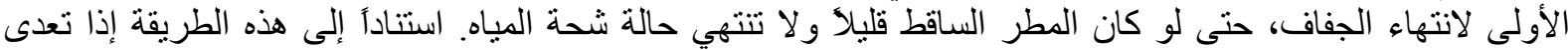

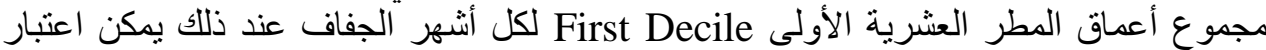

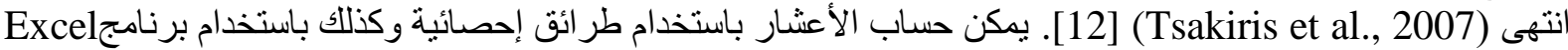

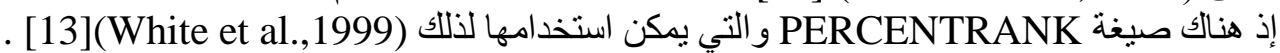


من المهم أن نفهم أن هذه الطريقة على الرغم من أنها أداة مفيدة عند دراسة التغير المناخي، ولكنها عملياً لا يمكنها

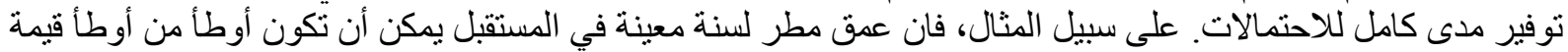

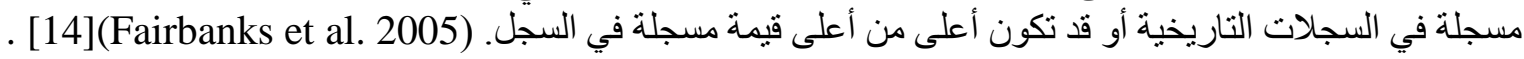

\section{النتائج والمناقشة}

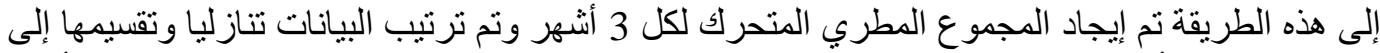

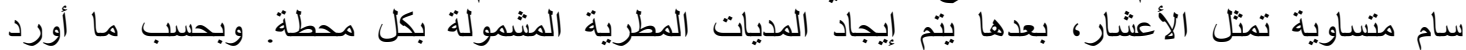

3 أثنهر يقع ضمن العشرية الثانية

[1] [11:(Kininmonth et al., 2000)

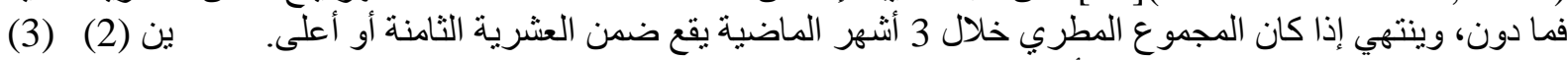

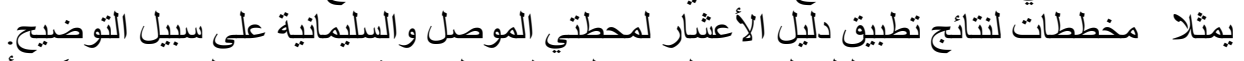

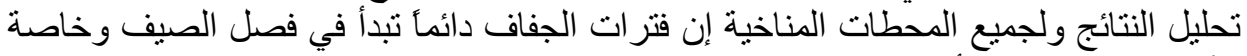

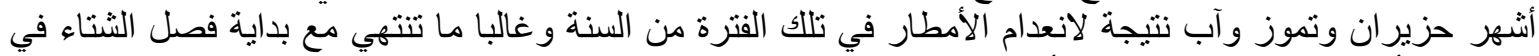

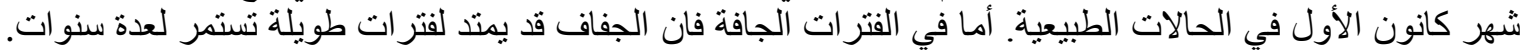

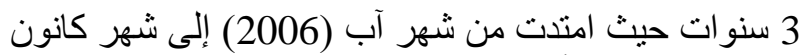

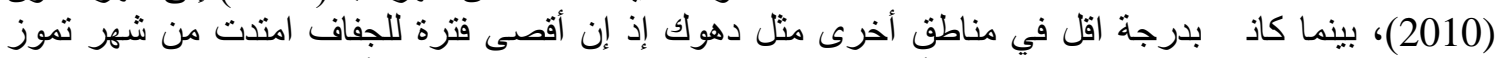

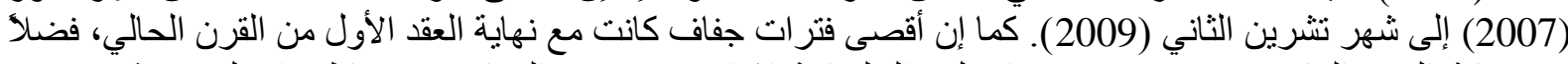

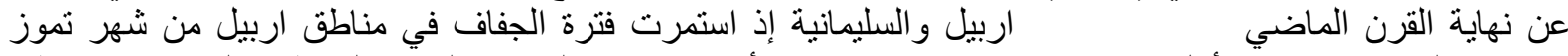

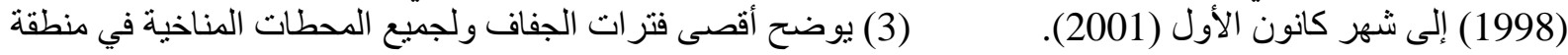

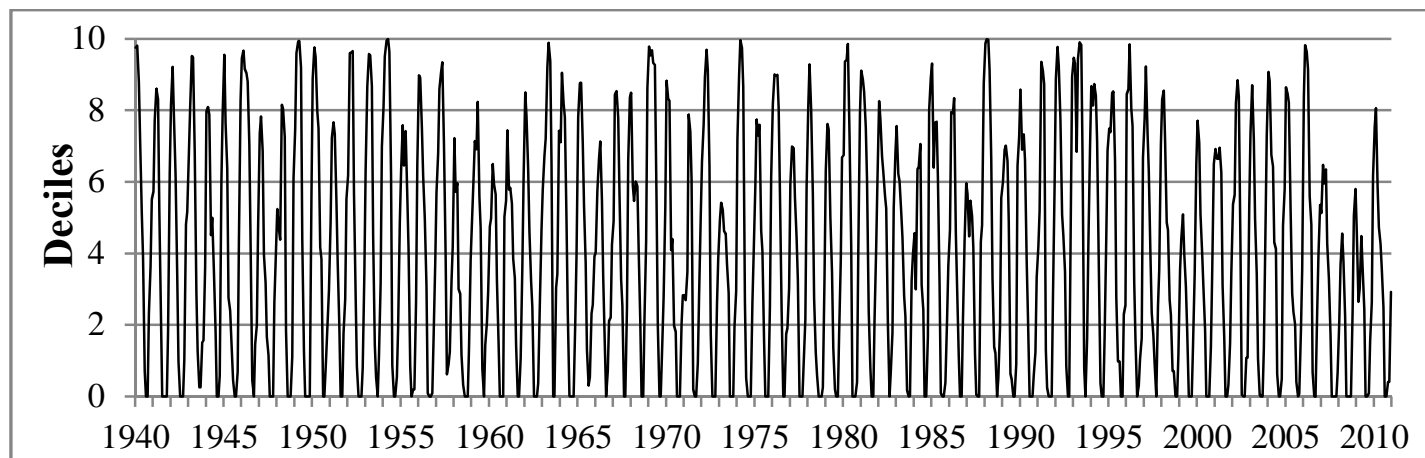

Time (Months)

(2): قيم المجموع المطري المتحرك لـ 3 أثهر ونوزيعها بحسب تصنيف الأعشار لمحطة أرصاد الموصل

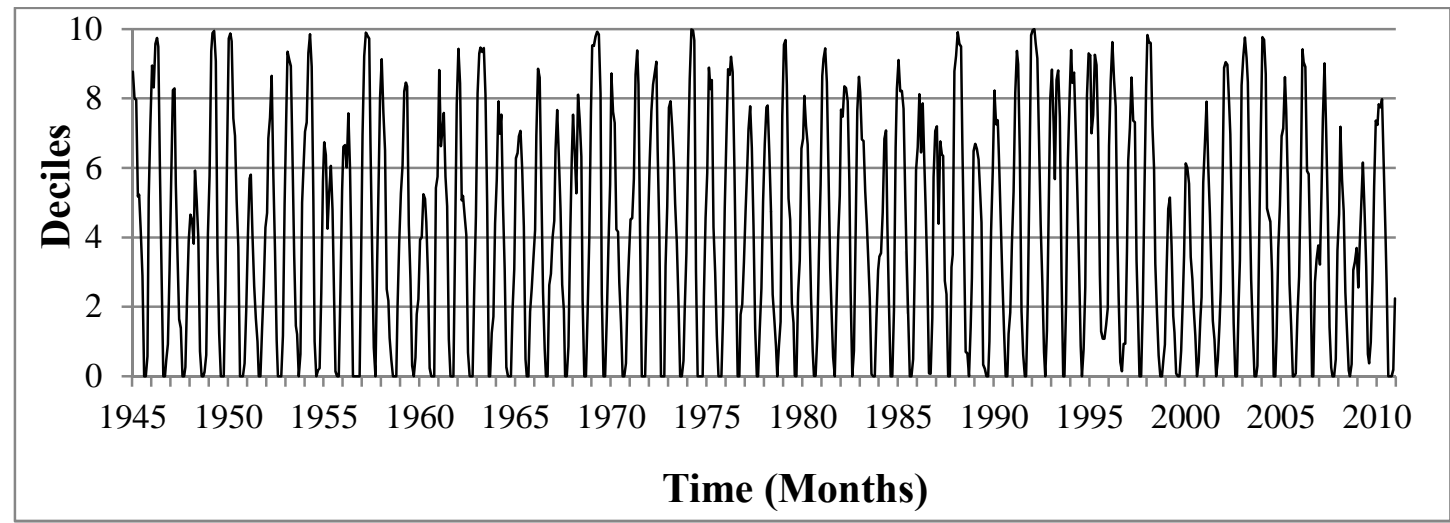

(3): قيم المجموع المطري المتحرك لـ 3 أثهر وتوزيعها بحسب تصنيف الأعشار لمحطة أرصاد السليمانية 
(3): أقصى فترات الجفاف التي تعرضت لها المنطقة مقاسة باستخدام دليل الأعشار (Deciles)

\begin{tabular}{|c|c|c|c|c|}
\hline \multirow[t]{2}{*}{ عدد أشهر الجفاف } & \multicolumn{2}{|c|}{ فترة الجفاف } & \multirow[t]{2}{*}{ المحطات المناخية } & \multirow[t]{2}{*}{ ت } \\
\hline & إلى & من & & \\
\hline 53 & 2010 & 2006 & & 1 \\
\hline 53 & 2010 & 2006 & & 2 \\
\hline 43 & 2010 & 2006 & & 3 \\
\hline 43 & 2010 & 2006 & & 4 \\
\hline 43 & 2001 & 1998 & دربنديخان & 5 \\
\hline 42 & 2001 & 1998 & اربيل & 6 \\
\hline 42 & 2009 & 2006 & & 7 \\
\hline 31 & 2001 & 1998 & السليمانية & 8 \\
\hline 29 & تشرين الثاني 2009 & 2007 & دهوك & 9 \\
\hline
\end{tabular}

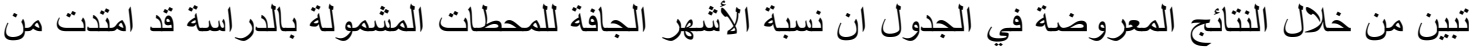

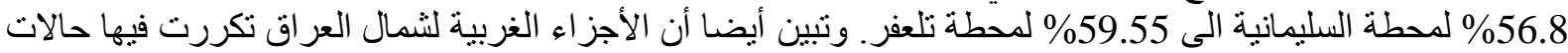

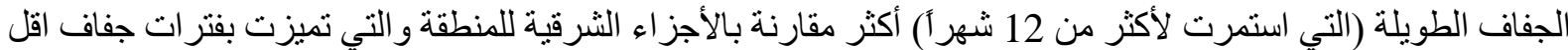

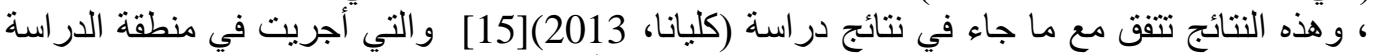

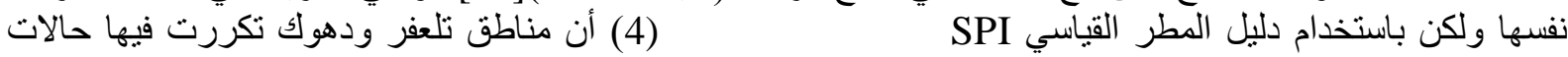
20.4

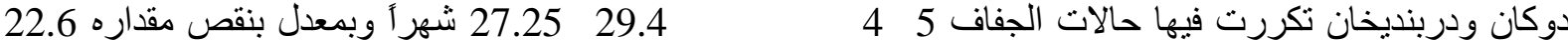

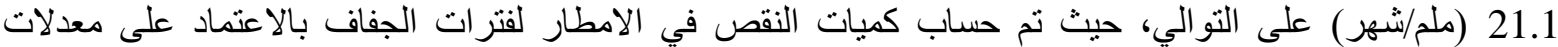

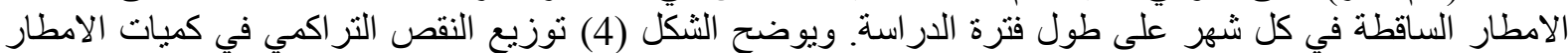

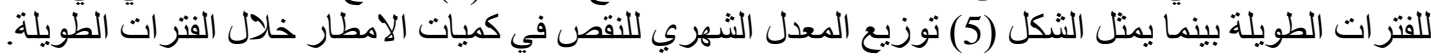

(4): معدلات فتر ات الجفاف وكميات النقص في السقيط للمحطات المشمولة بالدر اسة

\begin{tabular}{|c|c|c|c|c|c|c|c|c|c|}
\hline (ملم/شهر) & الطويلة ( ) & الطويلة & الطويلة* & (شهر ) & & شهر & أشهر & (شهر) & \\
\hline 9.4 & 191 & 20.4 & 10 & 8.6 & 58 & $\% 59.55$ & 499 & 838 & \\
\hline 13.7 & 256 & 18.7 & 10 & 8.28 & 57 & $\% 57.99$ & 472 & 814 & دهوك \\
\hline 10.8 & 241 & 22.3 & 9 & 8.61 & 57 & $\% 58.59$ & 491 & 838 & \\
\hline 14.1 & 333 & 23.6 & 8 & 8.46 & 59 & $\% 58.71$ & 499 & 850 & \\
\hline 9.3 & 197 & 21.3 & 7 & 7.88 & 65 & $\% 57.79$ & 512 & 886 & \\
\hline 17.2 & 353 & 20.6 & 7 & 7.68 & 62 & $\% 56.80$ & 476 & 838 & السليمانية \\
\hline 2.4 & 62 & 25.3 & 6 & 8.17 & 60 & $\% 58.47$ & 490 & 838 & اربيل \\
\hline 22.6 & 665 & 29.4 & 5 & 8.11 & 56 & $\% 57.47$ & 454 & 790 & \\
\hline 21.1 & 574 & 27.3 & 4 & 7.93 & 42 & $\% 56.83$ & 333 & 586 & دربنديخان \\
\hline
\end{tabular}


و استتاداً إلى ذلك فانه يمكن الإفادة من هذه الطريقة في إيجاد بداية ونهاية فترات الجنات الجفاف مستقبلا، فعلى سبيل المثال

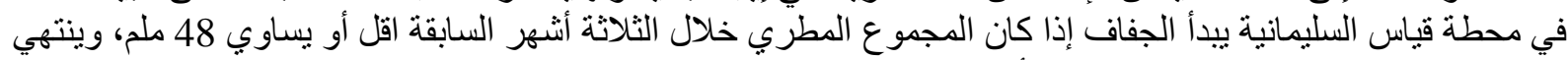

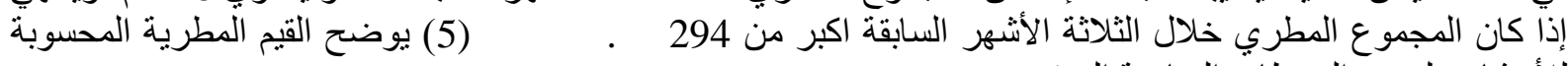
للأعثار ولجميع المحطات المناخية المعتمدة.

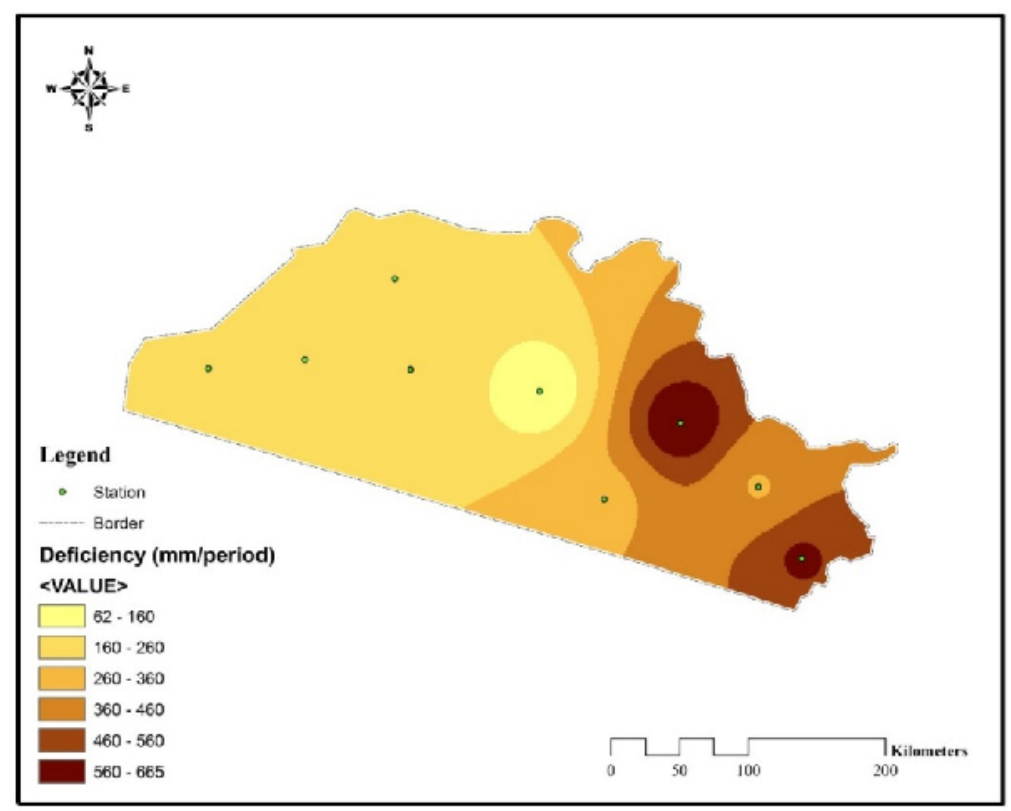

(4): توزيع مجموع النقص في كميات الامطار لفترات الجفاف التي تجاوزت 12 شهر آ ضمن منطقة الدراسة

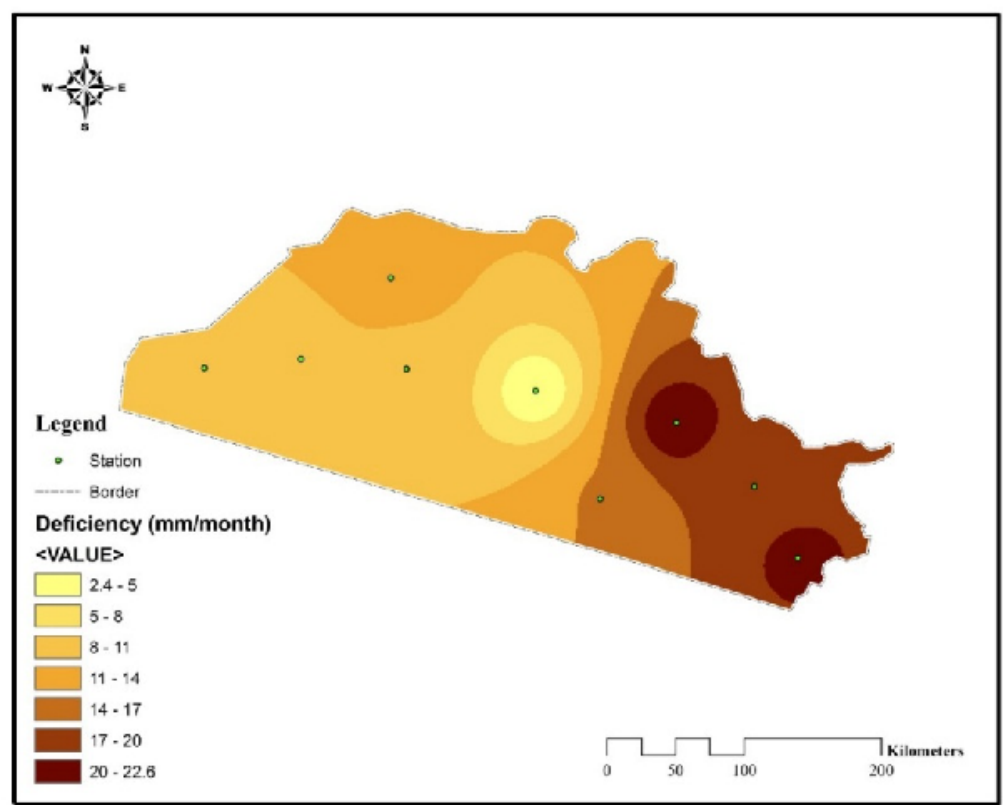

(5): المعدل الثهري للنقص في كميات الامطار للفترات التي تجاوزت 12 شهر أ ضمن منطقة الدر اسة 


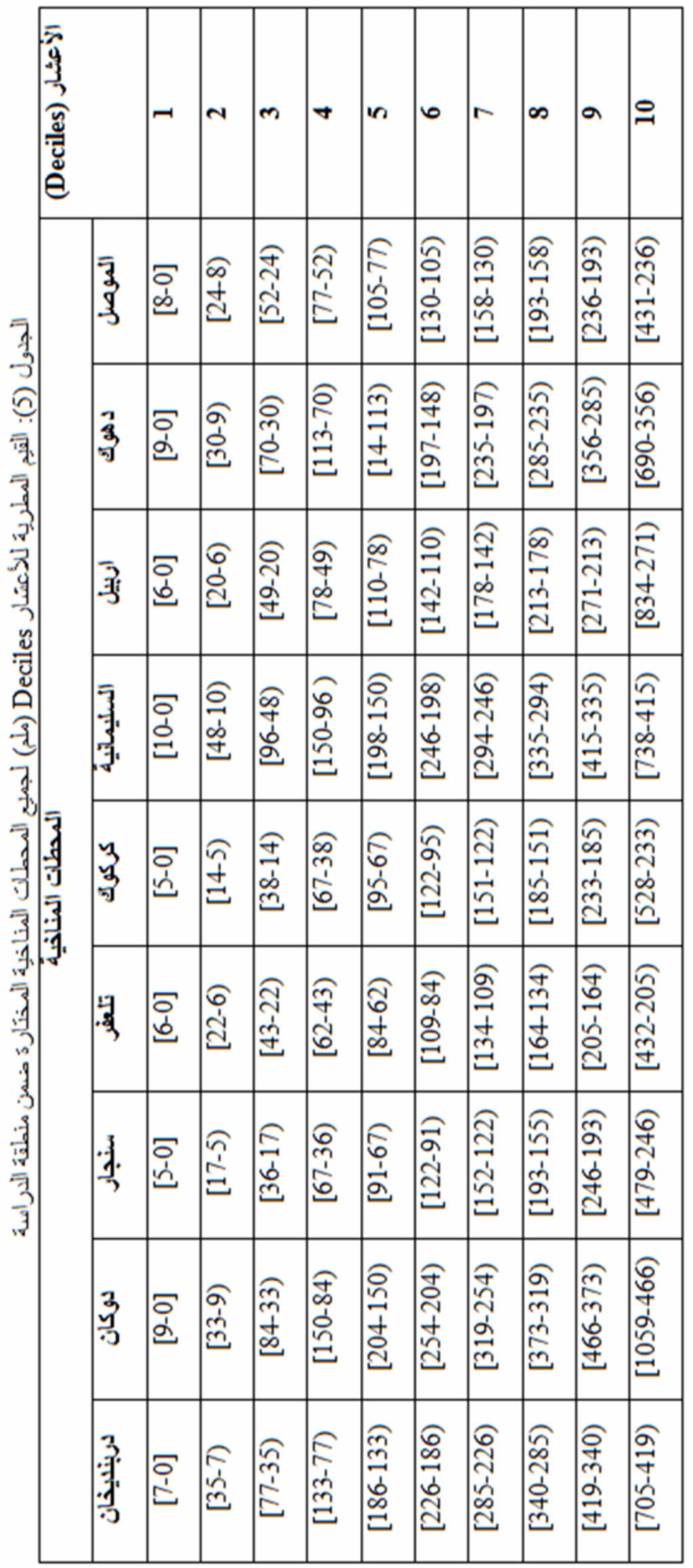




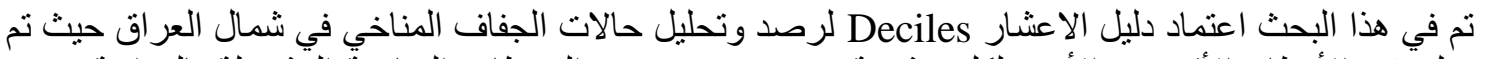

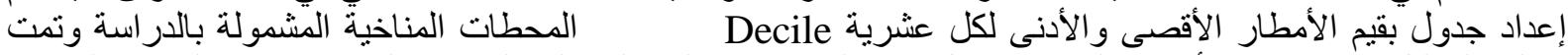

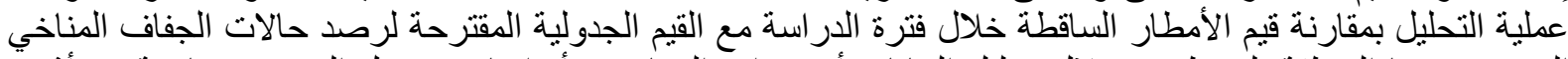

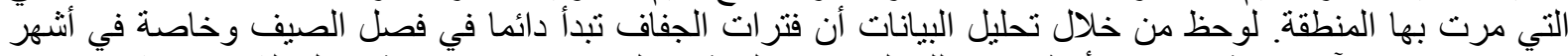

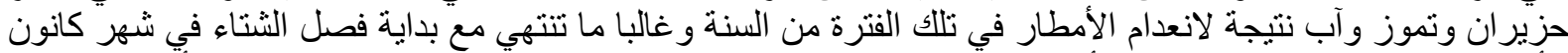

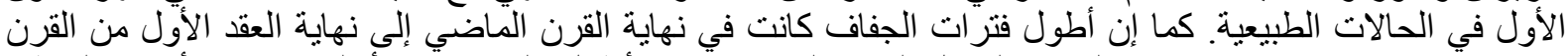

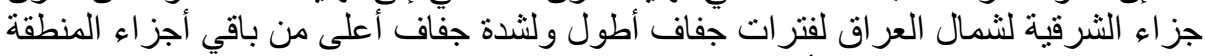

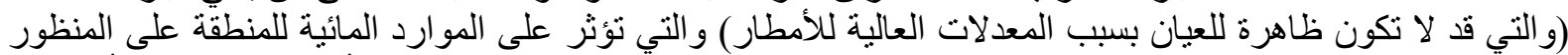

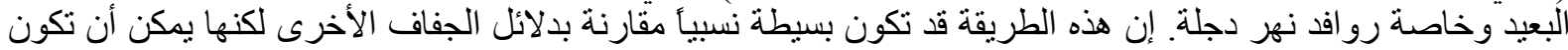

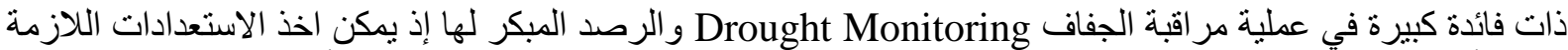

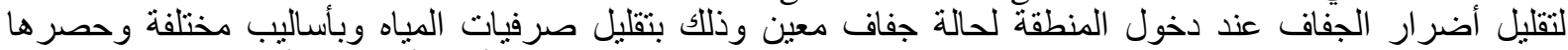

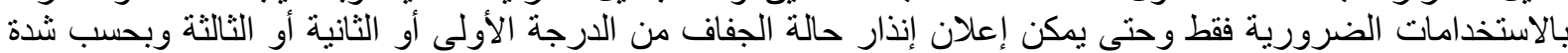
الجفاف التي تمر بها المنطقة وكما هو معدول به في الكثير من الدول المنار المنقدمة في العالم.

1. Perez, E., and Thompson, P., Natural Hazards: Drought (1995).

2. Smakhtin, V. U., and Hughes, D. A., "Review, automated estimation and analysis of drought indices in South Asia", Working Paper 83, Colombo, SriLanka, International Water Management Institute (2004).

3. Wilhite, D. A., "Drought as a natural hazard: Concepts and definitions", in D. A. Wilhite (ed.), Hazards disasters series, Routledge, New York, USA, 1: 3-18 (2000).

4. White, D. H., and O'Meagher, B., "Coping with exceptional droughts in Australia", Drought Network News, 7 (2): 13-17 (1995).

5. Tsakiris, G., Pangalou, D., and Vangelis, H., "Regional Drought Assessment Based on the Reconnaissance Drought Index (RDI)", Water Resources Management, 21: 821-833 (2006).

6. Timbal, B., "Observed climate changes in the south-east of Australia: detection and possible attribution", Hydrological Consequences of Climate Change, Canberra, Australia(2007).

7. Barua, S. "Drought Assessment and Forecasting Using a Nonlinear Aggregated Drought Index" a Ph.D. Thesis submitted to the Victoria University, Australia (2010)

8. Varshney, R. S., Gupta, S. C., and Gupta, R. L., "Theory and Design of Irrigation Structures; Volume 1, Channels and Tubewells", Fourth Edition, Roorkee Press, India (1979).

9. Gibbs, W. J., and Maher, J. V., "Rainfall deciles as drought indicators", Bureau of Meteorology Bulletin 48, Commonwealth of Australia, Melbourne (1967).

10.Smith, D. I., Hutchinson, M. F., and McArthur, R. J., “Australian climatic andagricultural drought: Payments and policy”, Drought Network News, 5 (3): 11-12 (1993).

11.Kinninmonth, W. R., Voice, M. E., Beard, G. S., de Hoedt, G. C., and Mullen, C. E., "Australian climate services for drought management", In: Drought: A Global Assessment, D. A. Wilhite (Ed.), Routledge, 210-222 (2000).

12.Tsakiris, G., Loukas, A., Pangalou, D., Vangelis, H., Tigkas, D., Rossi, G., and Cancelliere, A., "Drought characterization”, In: A. Iglesias, M. Moneo, A. Lopez-Francos (Eds) Drought Management Guidelines Technical Annex, Option Méditerranéennes 58, 85-102 (2007). 
13. White, I., Falkland, and Scott, D., "Droughts in small coral islands: Case study, South Tarawa, Kiribati”, UNESCO-IHP, Technical documents in hydrology No. 26 (1999).

14.Fairbanks, M., Tennant, D., Dracup, M., Beard, D., and Cramb, J., "What are rainfall deciles?",Farmnote 56, Department of Agricultural and Food, Government of Western Australia (2005) . www. agric. wa. gov.au

15- كليانا ، معد ماجد " نمذجة وتحليل الجفاف في شمال العراق" رسالة ماجستير مقدمة الى قسم هندسة السدود و الموارد المائية ، كلية الهندسة ، جامعة الموصل، لمدل (2013).

تم اجراء البحث في كلية ألهندسة = جامعة ألموصل 
آوجي: التحري عن الجفاف المناخي في شمال العراق باستخام طريقة الأعشار 